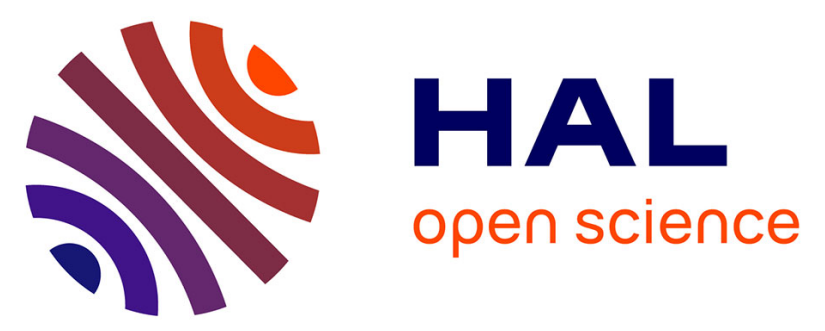

\title{
A Smooth Position-Force Controller for Asbestos Removal Manipulator
}

Kamal Mohy El Dine, Juan Antonio Corrales Ramon, Youcef Mezouar, Jean-Christophe Fauroux

\section{- To cite this version:}

Kamal Mohy El Dine, Juan Antonio Corrales Ramon, Youcef Mezouar, Jean-Christophe Fauroux. A Smooth Position-Force Controller for Asbestos Removal Manipulator. 2017 IEEE International Conference on Robotics and Biomimetics (ROBIO), Dec 2017, Macau, France. 10.1109/ROBIO.2017.8324599 . hal-01753927

\section{HAL Id: hal-01753927 https://hal.uca.fr/hal-01753927}

Submitted on 29 Mar 2018

HAL is a multi-disciplinary open access archive for the deposit and dissemination of scientific research documents, whether they are published or not. The documents may come from teaching and research institutions in France or abroad, or from public or private research centers.
L'archive ouverte pluridisciplinaire HAL, est destinée au dépôt et à la diffusion de documents scientifiques de niveau recherche, publiés ou non, émanant des établissements d'enseignement et de recherche français ou étrangers, des laboratoires publics ou privés. 


\title{
A Smooth Position-Force Controller for Asbestos Removal Manipulator
}

\author{
Kamal Mohy El Dine*, Juan-Antonio Corrales-Ramon ${ }^{\dagger}$, Youcef Mezouar ${ }^{\ddagger}$, and Jean-Christophe Fauroux ${ }^{\S}$ \\ Institut Pascal, SIGMA Clermont, Université Clermont Auvergne, CNRS, F-63000 ClermontFerrand, France \\ Emails:*Kamal.Mohy_El_Dine@ sigma-clermont.fr, †Juan-Antonio.Corrales-Ramon@ sigma-clermont.fr, \\ †Youcef.Mezouar@sigma-clermont.fr, ${ }^{\S}$ Jean-Christophe.Fauroux@ @igma-clermont.fr
}

\begin{abstract}
In robotic grinding tasks, the robot controller must reactively adapt to sudden changes in the environment and be able to handle uncertainties in texture, change in materials, and disturbances caused by vibrations, impacts and friction of the grinding tool operating at high rotational speed. This requires managing sudden signal changes in sensor data. In this paper, we present a smooth hybrid force/position controller based on distance measurements from radars, enabling the robot to achieve a stable interaction with the environment while grinding an unknown three-dimensional surface. The control uses an actively compliant wrist that maintains a desired force centered on the disc and normal to the surface. Our controller is based on a smooth transition between free space and contact modes, significantly reducing the impact force. Additionally, the vibro-dynamic effects are suppressed and smooth environmental tracking is ensured by the impedance/admittance control of the wrist. This framework is validated on a 6-dof anthropomorphic arm through dynamic simulation. The controller is able to adapt reactively to abrupt disturbances in the environment (ex: sudden impacts on the disc) while ensuring good position and force tracking performance.
\end{abstract}

\section{INTRODUCTION}

Despite the advents in industrial automation, robotic solutions are not yet commonly used in construction and demolition industries. However, implementation of robots in civil engineering is strongly growing, as the International Federation of Robotics (IFR) survey showed about 568 construction and demolition systems were supplied in 2015, and the number is estimated to increase to 2,800 in 2019 [1]. Currently, most tasks are performed manually with the help of conventional electro-mechanic and hydraulic tools. For example, asbestos removal by grinding is still performed by human operators, subjecting them to health hazards like cancer, since the asbestos fibers can infiltrate into respiratory system even with the use of protection. Additionally, the cleaning productivity is limited by human performance while the surface area of contaminated flats is considerable. Thus, the automation of this task to achieve efficient asbestos removal from a real world rehabilitation site, without endangering the human life is recommended [2], [3]. A conceptual design of the system can be represented as shown in Figure 1. In order to develop such system, investigation is carried out to build a multimodal control based on the association between radar and force informations that ensure a constant grinding force of desired magnitude and orientation. For this grinding task, the challenge is to achieve a smooth cleaning of the surface while

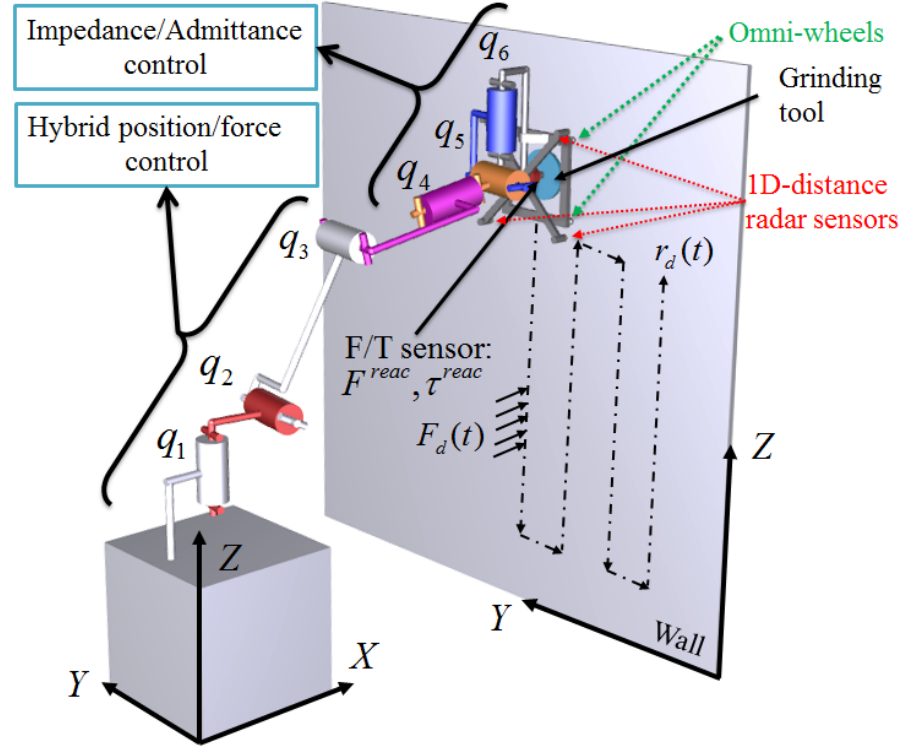

Fig. 1: Kinematic structure of the robotic system equipped with a grinding tool, several 1D-distance radar sensors around it for attitude measurement, and a force/torque sensor to perform asbestos removal on the wall

adapting to it and to the random impacts from the large grinding disc where the latter can not be neglected. The issues mentioned direct the problem into two main tracks: control strategies for robot-environment interaction and robotic grinding. Concerning the interaction with the environment, many important works have been carried out in the literature. The control strategies proposed can be grouped into two categories: indirect and direct force control, in which the former achieves motion control without explicit closure of a force feedback loop while the latter closes the force loop on the measured force value [4]. To the first category belong impedance and its alternatives, explained by Hogan in [5], where he introduced a method for a manipulator to interact dynamically with its environment. A meaningful set of controllers and their respective design specifications to assure compliant motion with stability robustness were described and analyzed in [6], [7] and a formalism for specifying compliant motion tasks and tracking was explained in [8]. The capabilities of impedance control have been enhanced by providing means of force 
tracking to keep a desired contact force with the environment despite lacking the knowledge of its stiffness and position [9]. This framework has been proposed by two simple on-line schemes. The first is based on generating the reference position on-line as a function of the force-tracking error and the second estimates environmental parameters using an indirect adaptive approach. Moreover, a force limited impedance and position limited force control were proposed in [10] by implementing an impedance controller as an inner loop and integral force controller as an outer loop. This allowed changing the behavior based on the limited external force. Hence, a good position, impedance and force tracking is obtained. A more advanced force tracking impedance control based on adaptive methods has been proposed in [11]. It worked well for abrupt changes in the environmental stiffness. Then the author of [12] proposed a predictive force control that used a fuzzy scaling machine. The controller performance seems good but it needs the estimation of the environmental stiffness. In [13], [14] the controller is based on learning to reduce the error and energy and make the robot behave like a human while interacting with unknown environments. It showed that the robot is resistant to uncertainties after learning but off-line training is essential. Carrying on performance improvement, [15] includes the redundancy resolution in the controller and solves the interaction by allowing the end-effector to comply according to an impedance control law defined in the Cartesian space. More works have been done on performance issues of the impedance and regarding external forces as presented in [16]-[20] where the researchers combined impedance with extra vision and tactile sensors.

On the other hand, hybrid position/force falls within the direct force control category. When the manipulator is in contact with the environment, the end-effector coordinate space can be decomposed into position and force subspaces, then control in each subspace can be done [21], [22]. The explicit model based hybrid control of rigid robot in contact with rigid environments has been studied in [21], [23], [24] and with compliant environments [25]-[27]. A review about the approaches used for controlling robots in constrained motions can be found in [28]. These hybrid controllers suffer from stability issues because of switching, impacts and uncertainties, which challenged researchers to investigate solutions. Attempts have been made to solve these issues using active stiffness and impedance control [29], [30]. Other solutions reduced environmental stiffness using mechanical means such as soft sensors or compliant covers [31], [32]. Passive and dynamic damping control was used [33]-[35] as an alternative.

As many studies deduced, using only one sensor limits the tasks the robot can do. Hence to ameliorate the force and position performances in unknown environments, the hybrid control is extended with vision [36]-[38]. The controllers suffered from disturbance and the closed loop stability was not proven. Then the uncertainties of the robot and the environment were taken into account and more work on the precision was done in [39]-[46]. Moreover, the contact problem has been addressed in the literature [37], [47], [48]. In [40], the force was added after contact to reduce impact, while in [42], the control switches from "position" before contact to "force" control after-wise. The author in [49] used motion control while approaching, followed by impact loading control that dissipates the impact force by setting the force command value negatively proportional to the velocity of the end-effector upon contact. In [50], the contact velocity is decreased based on vision and a rubber damper is added to reduce impact.

Vision is very useful for locating the environment, and determining the relative position and orientation of the robot's end-effector. It has been widely used to improve the humanrobot and robot-environment interactions. [51] shows how vision can be used to detect the system states to ensure safe interaction with the human. In [19], [45], [52], vision sensors were exploited in applications that require interaction with the environment. Although vision is powerful in canceling unwanted control behaviors and in adding the knowledge about environment, it is usually not sufficient when the environment is rugged and dusty. Hence, in Section II of this paper we present a unified control strategy based on 1-D radars, furnished with smooth transition function to minimize the impact force as it is explained in Subsection II-C. The grinding forces similar to the ones analyzed in [53] are limited by the controller. A detailed model is presented in Subsection II-F for grinding walls with abrasive disc, where it is used to validate the controller. Hence, taking the advantage of force measurements and radar information, better fitting to the surface can be achieved while grinding and ensuring a normal contact force on the wall coinciding with the tool center.

\section{CONTROL FRAMEWORK}

Without loosing generality, the control scheme presented in this paper can be applied to any robotic arm where position and orientation can be decoupled. The first three joints of the arm are used to ensure a desired force on the wall and trajectory tracking using hybrid position-force control. The control of wrist joints is based on admittance to ensure the adaptation of the tool to the wall as shown in Figure 1. The control scheme is explained in the following subsections:

\section{A. Hybrid Control in Operational Space}

In complex and unpredictable environments, e.g grinding operation, the end-effector motion may be subjected to sudden disturbances occurring due to excessive forces. Thus, in order to accommodate these unexpected interactions, the endeffector motion must be adapted by on-line modifications. Expressing the task in operational space requires a precise control of the end-effector motion, which can be achieved by the hybrid control proposed in [4]. Based on dynamics, the controller can be expressed as follows:

$$
h_{c}=\Lambda(q) S_{v} \alpha_{v}+S_{f} F_{c m d}+\mu(q, \dot{q})
$$

where $h_{c} \in R^{n}$ (applied on the $1^{s t} 3$ joints and $n=3$ ) denotes the output wrench of the end-effector in operational space; $q$ corresponds to joint values, $S_{v}$ and $S_{f}$ are the selection matrices of position and force controlled directions respectively; $\alpha_{v}$ 


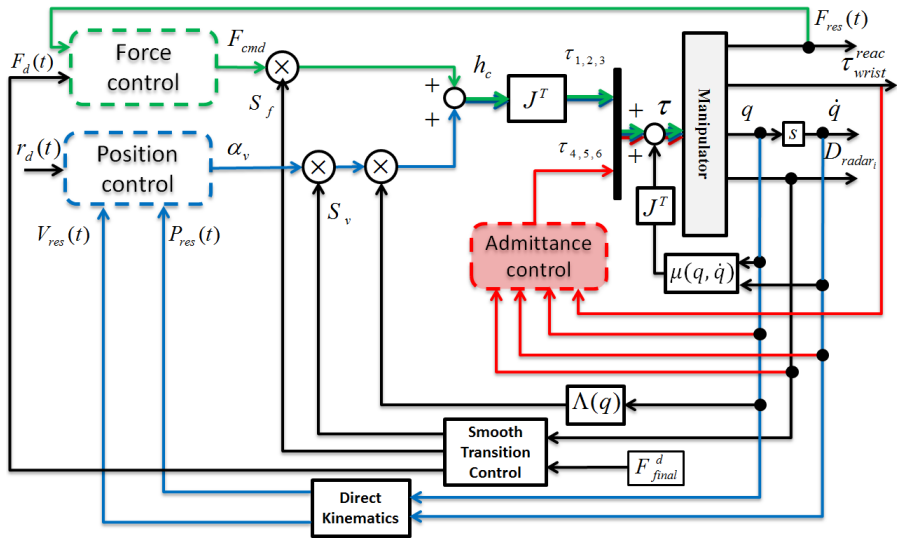

Fig. 2: Hybrid controller block diagram: force control loop (green), position control loop (blue), admittance control loop (red) (refer to Section II)

and $F_{c m d}$ are the acceleration and force commands respectively, $\Lambda(q)$ is the pseudo-inertia matrix defined by:

$$
\Lambda(q)=J H(q)^{-1} J
$$

with $J$ denoting the $(n \times n)$ kinematic Jacobian matrix, $H$ is the $(n \times n)$ robot inertia matrix; $\mu$ is the $(n \times 1)$ function to compensate for Coriolis, gravitational and friction forces in the workspace. It is defined by:

$$
\mu(q, \dot{q})=\Gamma(q, \dot{q}) \dot{q}+\eta(q)
$$

where $\Gamma$ is the wrench mapping the centrifugal, Coriolis and friction effects $c(q, \dot{q})$ from joint space into operational space:

$$
\Gamma(q, \dot{q})=J^{-T} c(q, \dot{q}) J^{-1}-\Lambda(q) \dot{J} J^{-1}
$$

and $\eta$ is the wrench mapping the gravitational effects $g(q)$ from joint space into the operational space as:

$$
\eta(q)=J^{-T} g(q)
$$

Finally the joint torques $\tau$ can be calculated by:

$$
\tau=J^{T} h_{c}
$$

The control loop expressed in equation (1) allows full decoupling between the force and velocity controlled subspaces (refer to Figure 2).

\section{B. Force and Position Control Loops:}

The desired force $F_{d}$ can be achieved by setting:

$$
F_{c m d}=F_{d}(t)+K_{P F}\left[F_{d}(t)-F_{\text {res }}(t)\right]
$$

$F_{c m}$ is the command to force controller, $F_{r e s}$ is the reaction force value and $K_{P F}$ is a suitable positive-definite gain matrix. The proportional feedback is able to reduce the force error due to disturbance forces.

Position control can be achieved by setting:

$$
\alpha_{v}=\ddot{r}_{d}(t)+K_{D r}\left[\dot{r}_{d}(t)-V_{\text {res }}(t)\right]+K_{P r}\left[r_{d}(t)-P_{\text {res }}(t)\right]
$$

$V_{r e s}$ and $P_{r e s}$ are the velocity and position response of the endeffector computed by the direct kinematics; $K_{D r}$ and $K_{P r}$ are suitable gain matrices; $\ddot{r}_{d}(t), \dot{r}_{d}(t)$ and $r_{d}(t)$ are the desired acceleration, velocity and position tracking inputs, obtained from the trapezoidal trajectory generator with continuous acceleration as detailed in [54].

\section{Smooth Transition Control}

As described in Section I, the switching problem is of main concern. In order to avoid the discontinuous switching between the controllers, and to reduce the impact force, a new strategy is introduced to change the selection matrix element $S(i, j)$ corresponding to the desired direction of motion from 0 to 1 smoothly. This way, the controller inputs are continuous and the control scheme smoothly flips from full position to hybrid control according to the distance from the grinding tool to the wall $D_{\text {wall }}$ obtained from radar readings:

$$
D_{\text {wall }}=\min \left(D_{\text {radar }_{1}}, D_{\text {radar }_{2}}, D_{\text {radar }_{3}}, D_{\text {radar }_{4}}\right)
$$

Hence, exponential variation has been chosen for this issue as:

$$
\begin{aligned}
& S_{f}(i, j)=S_{f_{0}} e^{k_{a} * D_{\text {wall }}} \\
& S_{v}(i, j)=1-S_{f}(i, j)
\end{aligned}
$$

with

$$
\begin{gathered}
k_{a}=-\frac{\log \left(\frac{S_{f_{\text {final }}}}{S_{f_{0}}}\right)}{D_{\text {final }}}+\varepsilon \\
\varepsilon=D_{\text {wall }}^{\text {final }}-D_{\text {wall }}^{\text {initial }}
\end{gathered}
$$

$S_{f_{\text {final }}}$ is chosen as a small scalar close to $0, S_{f_{0}}=1$ and the impact control is regulated by $\varepsilon$ according to the distance range defined by $\left[D_{\text {wall }}^{\text {final }}-D_{\text {wall }}^{\text {initial }}\right]$. When $S_{f}(i, j)$ reaches $1, F_{d}$ starts from 0 to reach the maximum desired value:

$$
F_{d}(t)= \begin{cases}0 & \text { if } t \leq t_{\text {impact }} \\ r_{f}\left(t-t_{\text {impact }}\right)+F_{0} & \text { if } t_{\text {impact }}<t \leq t_{\text {impact }}+w \\ F_{\text {final }}^{d} & \text { if } t>t_{\text {impact }}+w\end{cases}
$$

where $r_{f}=\frac{F_{\text {final }}^{d}}{w}$ is the force rate, $F_{0}$ is the initial value of $F^{d}$ and $w$ is the desired period to reach the maximum force.

\section{Impedance/Admittance Based Orientation Control}

In order to ensure soft interaction with the environment and stability against impacts, joint based impedance/admittance controller has been utilized for the wrist joints [55]. It ensures the tool-wall adaption with minimal torques (refer to Figure 3 ). The torque acting on the end-effector when moving along the surface is:

$$
\tau_{\text {ext }}=K_{\text {reac }} \tau_{w r i s t}^{\text {reac }}
$$

$K_{\text {reac }}$ is gain of reaction forces, $\tau_{w r i s t}^{r e a c}$ is the reaction torque on the wrist, obtained from the torque sensor. Hence, the controller can be realized based on accelerations references as:

$$
\ddot{q}_{r e f}^{\text {total }}=\frac{1}{M_{i}}\left(\tau_{\text {ext }}-D_{i} \dot{q}_{r e f}^{\text {total }}-K_{i} q_{r e f}^{\text {total }}\right)
$$




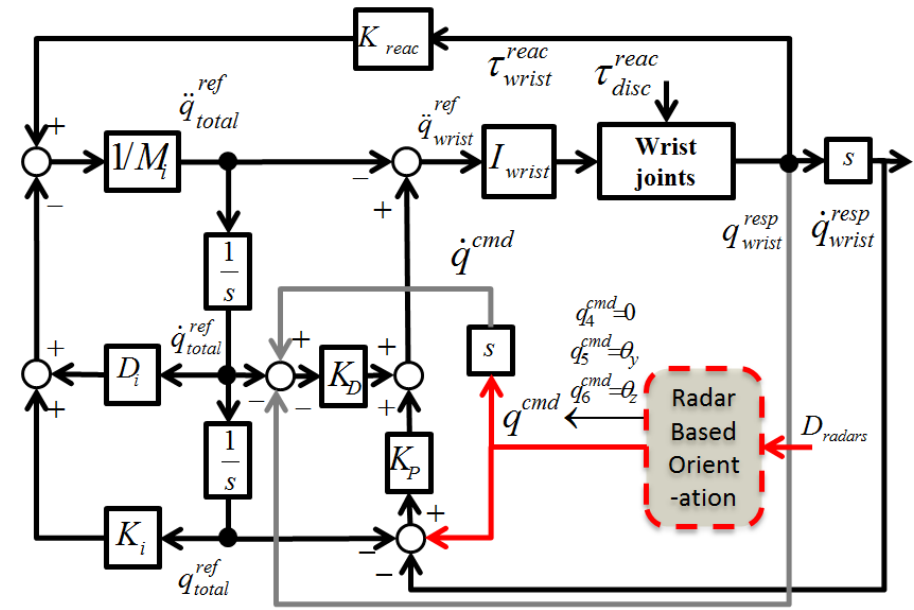

Fig. 3: Joint based admittance controller block diagram (refer to Sections II-D, II-E)

where $M_{i}, D_{i}$ and $K_{i}$ are the impedance gains. They can be tuned by fixing the $2^{\text {nd }}$ order dynamics as:

$$
\frac{\ddot{q}_{w r i s t}^{r e f}}{\tau_{\text {ext }}}=\frac{1}{M_{i} s^{2}+D_{i}+K_{i}}
$$

The natural frequency $\omega_{i}$ and the damping ratio $\zeta_{i}$ are defined as:

$$
\omega_{i}=\sqrt{\frac{K_{i}}{M_{i}}} \text { and } \zeta_{i}=\frac{D_{i}}{2 \sqrt{M_{i} K_{i}}}
$$

Finally, the motors angular acceleration can be defined as:

$$
\begin{aligned}
\ddot{q}_{\text {wrist }}^{\text {ref }}=-\ddot{q}_{\text {ref }}^{\text {total }}+ & K_{p}\left(q^{\text {cmd }}-q_{\text {ref }}^{\text {total }}-q_{\text {resp }}^{\text {wrist }}\right) \\
& +K_{d}\left(\dot{q}^{\text {cmd }}-\dot{q}_{\text {ref }}^{\text {total }}-\dot{q}_{\text {resp }}^{\text {wrist }}\right)
\end{aligned}
$$

and the torque for wrist joint as,

$$
\tau_{w r i s t}^{r e f}=I_{w r i s t} \ddot{q}_{w r i s t}^{r e f}
$$

where $K_{p}$ and $K_{d}$ are the PD gains respectively, and $I_{w r i s t}$ is the wrist inertia matrix.

\section{E. Radar Based Orientation}

Based on radar measurements, the absolute rotations between the tool and the wall, shown in Figure 4 can be calculated as:

$$
\left\{\begin{array}{l}
\theta_{y}=\operatorname{sign}\left(r_{3}-r_{2}\right)\left(\frac{\pi}{2}-\gamma\right) \\
\theta_{z}=\operatorname{sign}\left(r_{1}-r_{2}\right)\left(\frac{\pi}{2}-\alpha\right)
\end{array}\right.
$$

With

$$
\begin{gathered}
\gamma= \begin{cases}\tan ^{-1}\left(\frac{d_{r_{2} r_{3}}}{r_{2}-r_{3}}\right) & \text { if } r_{3} \leq r_{2} \\
\tan ^{-1}\left(\frac{d_{r_{2} r_{3}}}{r_{3}-r_{2}}\right) & \text { if } r_{3}>r_{2}\end{cases} \\
\alpha= \begin{cases}\tan ^{-1}\left(\frac{d_{r_{1} r_{2}}}{r_{2}-r_{1}}\right) & \text { if } r_{1} \leq r_{2} \\
\tan ^{-1}\left(\frac{d_{r_{1} r_{2}}}{r_{1}-r_{2}}\right) & \text { if } r_{1}>r_{2}\end{cases}
\end{gathered}
$$

$r_{1}, r_{2}$ and $r_{3}$ are the radar measurements respectively, and $d_{r_{i}} r_{j}$ is the distance between radars $i$ and $j$ (refer to Figure 4).

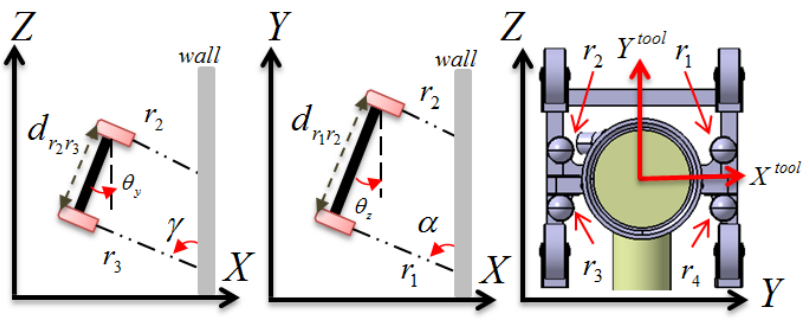

Fig. 4: Tool absolute orientation

TABLE I: Parameters used in grinding model.

\begin{tabular}{|c|c|c|}
\hline Variable & Unit & Explanation \\
\hline \hline$M R R$ & $\mathrm{~m}^{3} / s$ & Material removal rate \\
\hline$U$ & $J$ & Kinetic energy \\
\hline$\omega_{0}$ & $\mathrm{rad} / \mathrm{s}$ & Free rotational speed of the disc \\
\hline$F$ & $N$ & Applied force \\
\hline$m$ & $K g$ & Mass \\
\hline$t$ & $m$ & Thickness of the cut ahead \\
\hline$R_{1}, R_{2}, R_{3}$ & $m$ & Contact radii on the disc \\
\hline$d$ & $m$ & Depth of the cut \\
\hline$b$ & $m$ & Width of the cut \\
\hline$D$ & $m$ & Disc diameter \\
\hline$v$ & $m / s$ & End-effector velocity \\
\hline$a$ & $m / s^{2}$ & End-effector acceleration \\
\hline$A_{\text {contact }}$ & $m^{2}$ & Wall-disc contact area \\
\hline$K_{1}$ & $J . m$ & Constant relating the cut ahead to the energy \\
\hline$\beta$ & $\mathrm{rad} /(m . N . s)$ & Speed reduction to torque constant \\
\hline$\mu$ & & Friction constant \\
\hline$\delta$ & N.m & Delta function \\
\hline$n$ & N.m & Limited noise \\
\hline
\end{tabular}

\section{F. Disturbance Model of Grinding Torque}

In order to test the control response, and to simulate it against noises and impacts of the grinding process, a torque disturbance model is needed. In [53], a metal grinding process was modeled. The normal force on the disc was estimated based on it's physical parameters. Moreover, speed reduction value is formulated based on this normal force as:

$$
\text { speed reduction value }=\beta R \mu F_{\text {norm }}
$$

$R$ is the radius to the point of contact, the variables $\beta, \mu$ and $F_{n o r m}$ are defined in in Table I. In [56], the grinding force was estimated as a function of tool kinetic energy $(U)$, material removal rate (MRR) and the disc speed on the wall $\left(v_{\text {norm }}\right)$ as:

$$
F_{\text {norm }}=U * \frac{M R R}{v_{\text {norm }}}
$$

In our case, as the disc lies in plane of the wall, force exerted on the disc and it's speed are known. The interest here is to study the effect of grinding parameters defined in Table I and Figure 5 on the generation of the reaction torque. A model has been developed based on existing studies as:

$\tau_{\text {disc }}^{\text {reac }}=\operatorname{sign}\left(F_{\text {norm }}\right) * U *\left(\frac{(M R R)_{\text {tan }}+(M R R)_{\text {norm }}}{\omega_{0}-\omega_{\text {overload }}}\right)+N(n, \delta)$ 


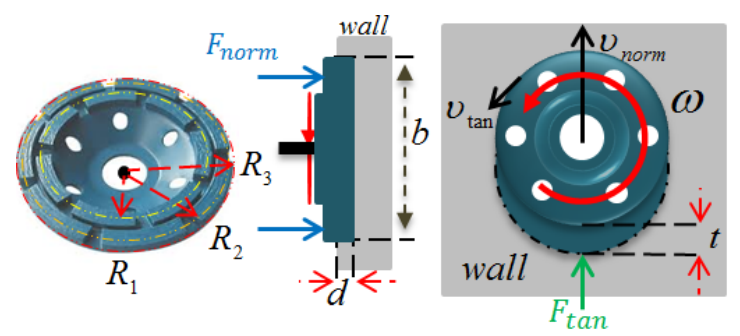

Fig. 5: Grinding parameters

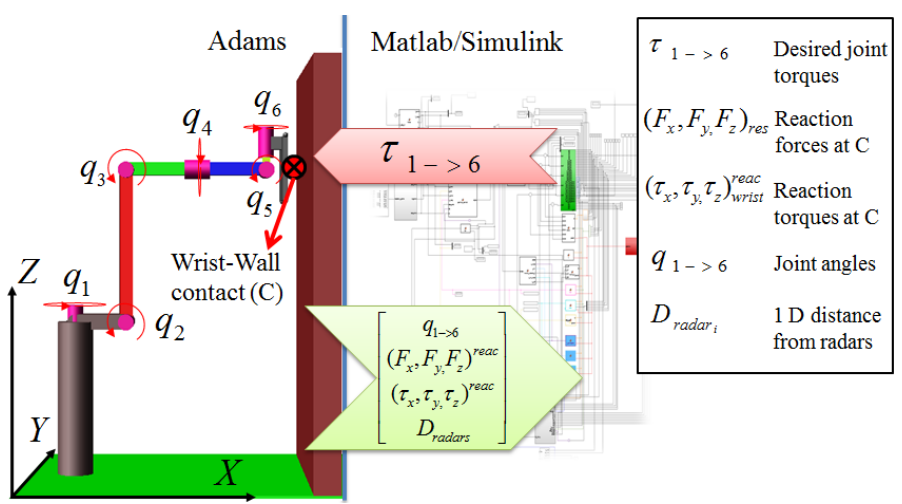

Fig. 6: Adams/Matlab co-simulation

with,

$$
\begin{cases}\omega_{\text {overload }} & =\beta \mu\left(R_{1} F_{\text {norm }}^{R_{1}}+R_{2} F_{\text {norm }}^{R_{2}}+R_{3} m a_{\text {tan }}\right) \\ (M R R)_{\text {tan }} & =v_{\text {tan }} d b \\ (M R R)_{\text {norm }} & =v_{\text {norm }} D A_{\text {contact }} \\ U & =K_{1} / t\end{cases}
$$

Where tan and norm subscripts stand for tangential and normal directions respectively. Additionally, random impacts $\delta$ and limited noise $n$ have been included in the noise function $N$ to represent a real case scenario.

\section{SIMULATIONS}

The control framework in Section II is implemented for a 6-R robot using Adams-Matlab co-simulation (Figure 6). The system is simulated with a trapezoidal trajectory generator that provides continuous acceleration in the variable velocity phases and constant speed otherwise. The robot is commanded to apply a force of $90 \mathrm{~N}$ on a desired path on the wall. It starts from free space and goes into the wall by a smooth transition from position to force control avoiding impact and maintaining the desired force. The admittance controller ensures centering the force and adapting to the wall.

\section{A. Hybrid Force-Position Control Behavior}

The command-response of trajectory position and velocity are shown in Figures 7 and 9 respectively. The controller shows good performance in free space and after contact, limiting the position errors to $0 \mathrm{~mm}$ before and $5 \mathrm{~mm}$ after contact at $t=1.4 \mathrm{~s}$ as shown in Figure 8. This variation in position errors is due to the $0.4 \mathrm{~N}$ dynamic friction force between

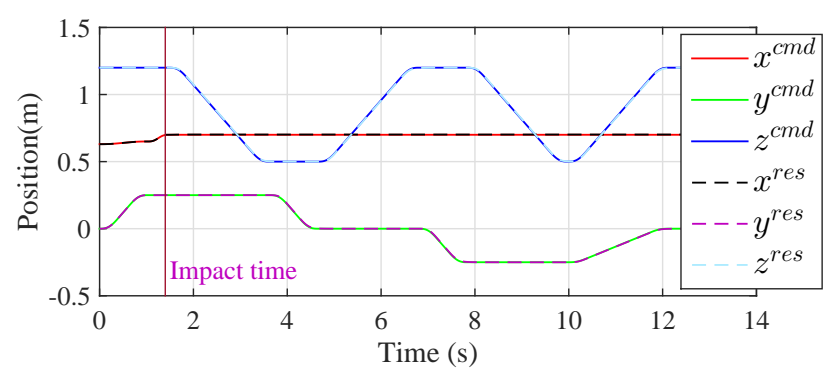

Fig. 7: Position command-response

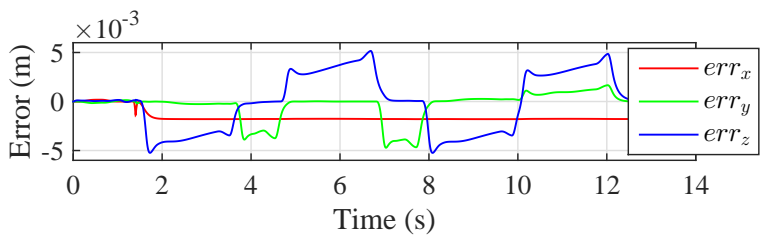

Fig. 8: Position command-response errors

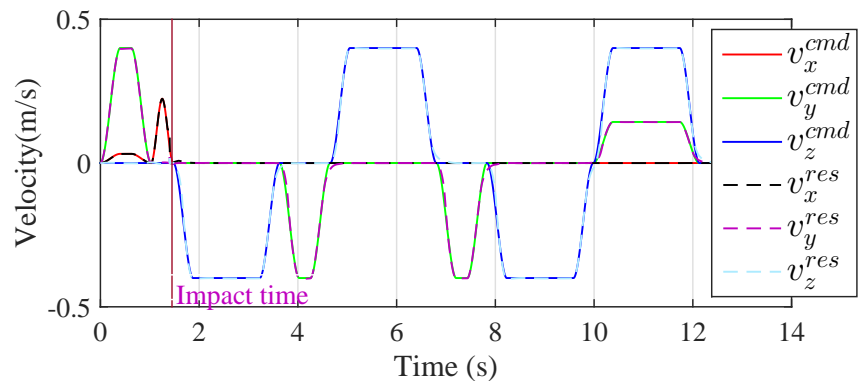

Fig. 9: Velocity command-response

the tool and wall, that is defined in Adams. Consequently the velocity errors are between $0.035 \mathrm{~m} / \mathrm{s}$ and $-0.05 \mathrm{~m} / \mathrm{s}$ after impact and are shown in Figure 10.

Concerning the force control performance, the force command versus response are plotted in Figure 11. The control with smooth transition in Figure 11b shows negligible impact force when touching the wall. Then the desired force reference value is reached in a behavior similar to a step function. The force value is maintained along the path with an error less than $2 \mathrm{~N}$ as shown in Figure 12b. The smooth transition control shows better values compared to the ones obtained by direct switching in Figure 11a, where the impact is clearly high and the force error can exceed $100 \mathrm{~N}$ as shown in 12a. Thanks to the smooth transition control presented in II-C that flips smoothly from position into force control in a unified manner that avoids switching as Figure 13 shows.

\section{B. Admittance Control Behavior}

As described in II-D, the wrist motors are based on acceleration control (eq.19) in addition to the torque sensor to measure the reaction torques $\tau_{w r i s t}^{\text {reac }}$. The controller gains $M_{i}, D_{i}$ and $K_{i}$ are tuned for $2^{\text {nd }}$ order differential equation to ensure stability and equivalent distribution of contact forces on the 


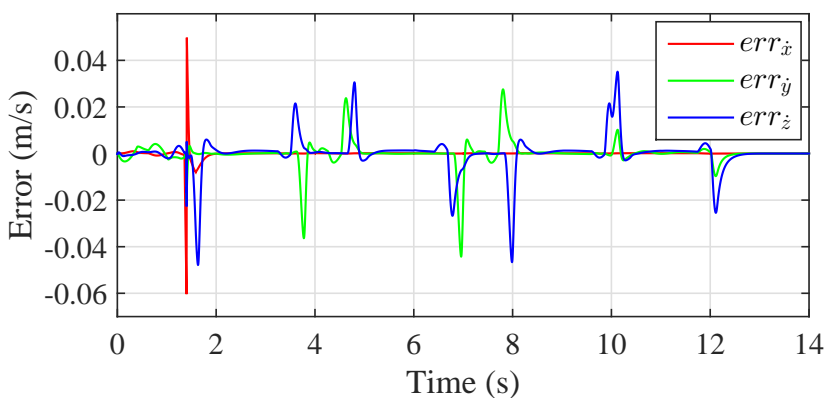

Fig. 10: Velocity command-response errors

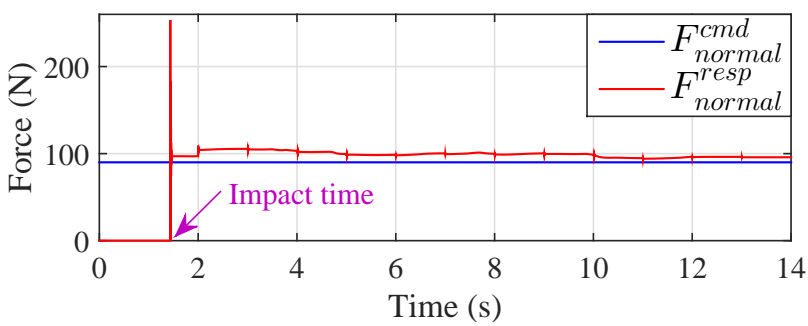

(a) Force command-response without smooth transition

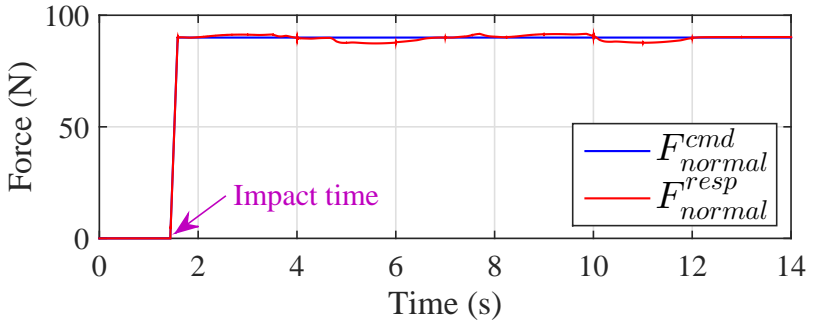

(b) Force command-response with smooth transition

Fig. 11: The command-response of normal force on the wall

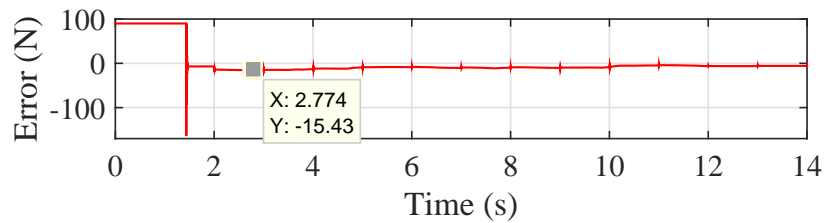

(a) Force command-response error without smooth transition

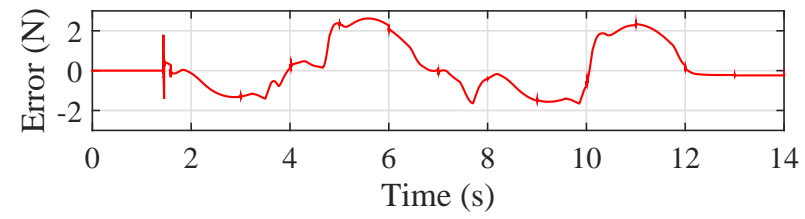

(b) Force command-response error with smooth transition

Fig. 12: Normal force command-response errors

end-effector (eq.17 \& 18). $K_{p}$ and $K_{d}$ are proportional and derivative gains tuned for good tracking of the reference values generated from commands and external torques (eq.16 \& 19) as shown in Figure 17, where the joint positions are modified based on external torques. The effectiveness of the admittance

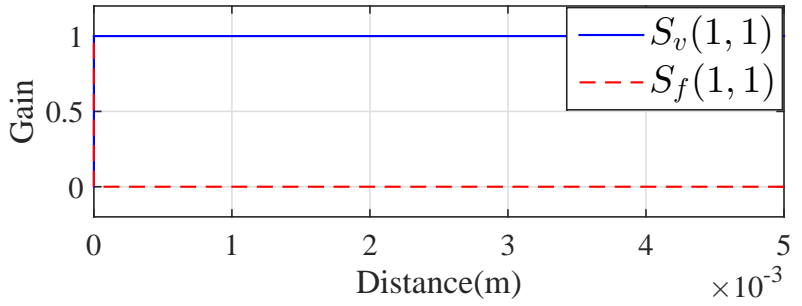

(a) Gains for direct switching from position to force control

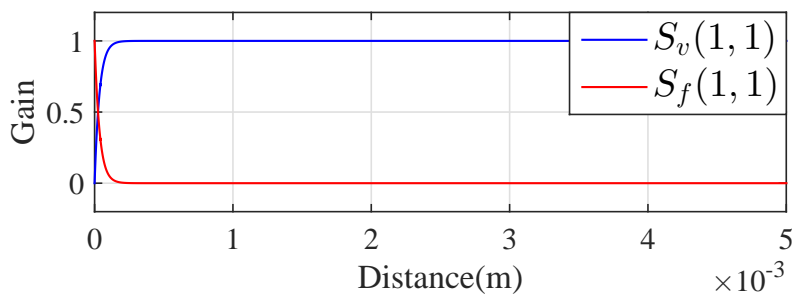

(b) Gains for smooth transition from position to force control

Fig. 13: Smooth switching from position to force control. $(1,1)$ is the index to the first element of the selection matrix

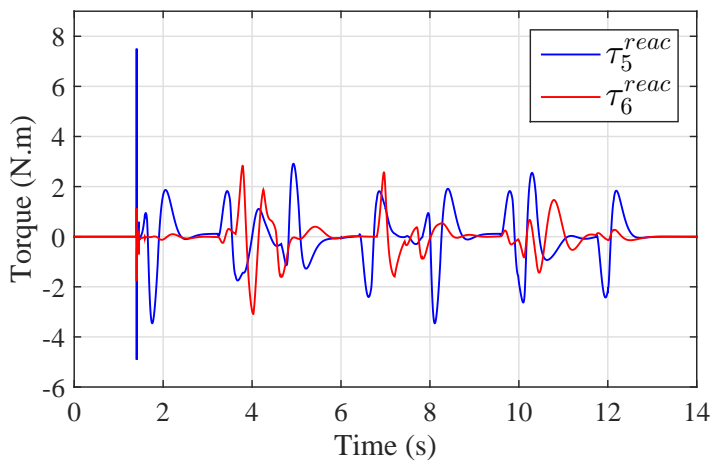

Fig. 14: The reaction torques on $5^{\text {th }}$ and $6^{\text {th }}$ joints

controller in adapting the tool to the wall, can be deduced from the fact of keeping minimal torques on $5^{\text {th }}$ and $6^{\text {th }}$ joints, that are responsible for the pitch and yaw of the tool as Figure 14 shows. Figure 15 shows how the controller maintains the force centered as the zero-moment-point of the tool-wall contact gets about the center of the tool (except for the first contact) overcoming lateral forces.

Adding external torque to the disc with some impacts, mainly results on the $4^{\text {th }}$ joint because of the mechanical linkage of the wrist that is similar to universal joint that couples rotation between two drive-line shafts: the base shaft (axle of the disc) and the follower shaft (joint 4). The results are shown in Figure 16 where the $\tau_{4}$ in 16a shows the behavior of joint 4 without external torque. However, when the end effector is subjected to external torque as in $16 \mathrm{~b}, \tau_{4}$ acts as shock absorber and it can suppress noise and impacts of the disc as 16c shows, thus avoiding high impacts on the joint. 


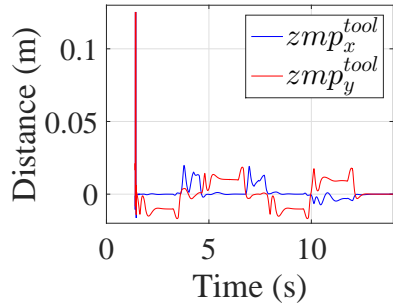

(a) Zmp coordinates

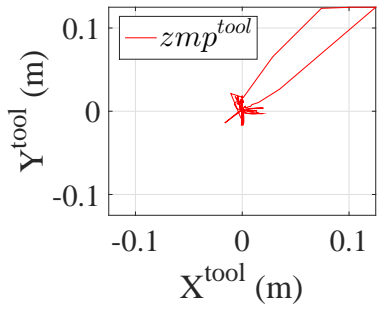

(b) Zmp trajectory
Fig. 15: Zero-moment-point on the end-effector expressed in the tool frame shown in Figure 4

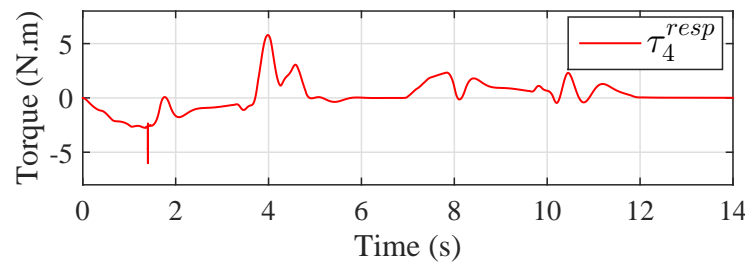

(a) Response of $\tau_{4}$ without the disc reaction torque

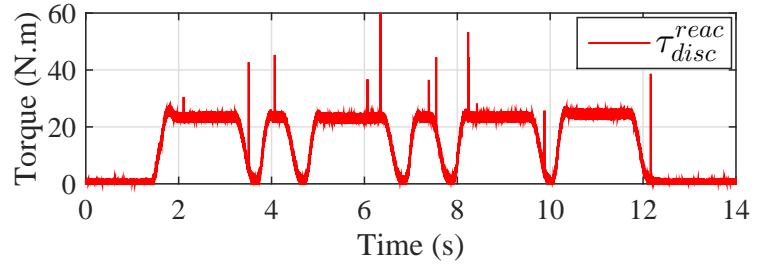

(b) Disc reaction torque plus noise and random impacts

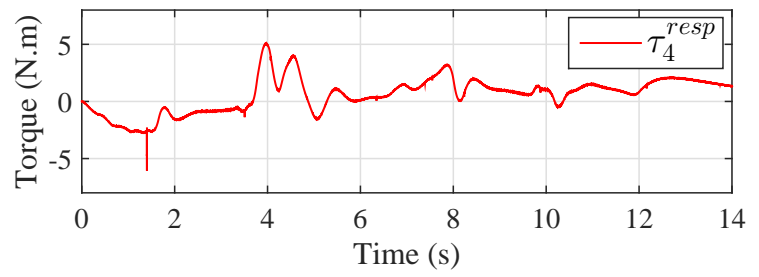

(c) Response of $\tau_{4}$ with the disc reaction torque added

Fig. 16: Suppressing the disc noise and random impacts by impedance control

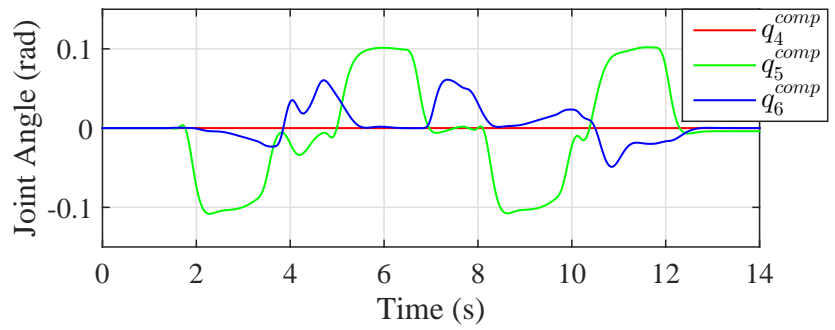

Fig. 17: Position compensation by admittance control

\section{CONCLUSIONS AND FUTURE WORK}

This article presents a smooth position-force hybrid controller that can reactively adapt to sudden changes in the environment and the resulting impacts while grinding. The controller is based on 1D-distance measurement radars and active admittance for tracking unknown three-dimensional surfaces while maintaining a desired force centered on the disc and normal to the surface. The switching problem is overcome by proposing a smooth transition control. The controller changes smoothly between free space and contact modes, thus reducing impact force close to zero and avoiding uncertainties. Additionally, a disturbance model to estimate the reaction torque of the tool has been developed in order to test the controller capable of suppressing vibro-dynamic effects. The control is validated by simulations on a 6-dof anthropomorphic arm. The results show good position and force tracking performances and impact force close to zero. In the near future, the controller will be implemented and tested by real robot on flat and curved surfaces in order to better evaluate and ameliorate its performance.

\section{ACKNOWLEDGMENT}

"The research leading to these results has received funding from the European Union's Horizon 2020 research and innovation programme under grant agreement No. 687593."

\section{REFERENCES}

[1] IFR, "Executive summary world robotics 2016 service robots," https://ifr.org/downloads/press/02_2016/Executive_Summary_Service_ Robots_2016.pdf, 2016, [Online; accessed 07-june-2017].

[2] S. Chazelet and E. Silvente, "Synthèse de la campagne INRS pour la détermination des facteurs de protection assignés des appareils de protection respiratoire utilisés en chantier de désamiantage," $\mathrm{Ph} . \mathrm{D}$. dissertation, Institut National de Recherche et de Sécurité (INRS), 2016.

[3] "Robots to Re-Construction," http://bots2rec.eu, 2015, [Online; accessed 07-june-2017].

[4] B. Siciliano and O. Khatib, Springer handbook of robotics. Springer, 2016.

[5] N. Hogan, "Impedance control: An approach to manipulation: Part iiimplementation," Journal of dynamic systems, measurement, and control, vol. 107, no. 1, pp. 8-16, 1985.

[6] H. Karerooni, T. Sheridan, and P. Houpt, "Robust compliant motion for manipulators, part i: The fundamental concepts of compliant motion, part ii: Design method," IEEE J. Robotics Automat, vol. 2, no. 2, pp. $83-105,1986$

[7] H. Kazerooni, "On the robot compliant motion control," Journal of dynamic systems, measurement, and control, vol. 111, no. 3, pp. 416425, 1989.

[8] J. De Schutter and H. Van Brussel, "Compliant robot motion i. a formalism for specifying compliant motion tasks," The International Journal of Robotics Research, vol. 7, no. 4, pp. 3-17, 1988.

[9] H. Seraji and R. Colbaugh, "Force tracking in impedance control," The International Journal of Robotics Research, vol. 16, no. 1, pp. 97-117, 1997.

[10] F. Almeida, A. Lopes, and P. Abreu, "Force-impedance control: a new control strategy of robotic manipulators," Recent advances in Mechatronics, pp. 126-137, 1999.

[11] S. Jung, T. C. Hsia, and R. G. Bonitz, "Force tracking impedance control of robot manipulators under unknown environment," IEEE Transactions on Control Systems Technology, vol. 12, no. 3, pp. 474-483, 2004.

[12] L. Baptista, J. Sousa, and J. S. da Costa, "Predictive force control of robot manipulators in nonrigid environments," from Industrial Robotics: Theory, Modeling and Control, pp. 841-874, 2006.

[13] G. Ganesh, A. Albu-Schäffer, M. Haruno, M. Kawato, and E. Burdet, "Biomimetic motor behavior for simultaneous adaptation of force, impedance and trajectory in interaction tasks," in Robotics and Automation (ICRA), 2010 IEEE International Conference on. IEEE, 2010, pp. $2705-2711$. 
[14] C. Yang, G. Ganesh, S. Haddadin, S. Parusel, A. Albu-Schaeffer, and E. Burdet, "Human-like adaptation of force and impedance in stable and unstable interactions," IEEE transactions on robotics, vol. 27, no. 5, pp. 918-930, 2011.

[15] F. Ficuciello, A. Romano, L. Villani, and B. Siciliano, "Cartesian impedance control of redundant manipulators for human-robot comanipulation," in 2014 IEEE/RSJ International Conference on Intelligent Robots and Systems. IEEE, 2014, pp. 2120-2125.

[16] G. Morel, E. Malis, and S. Boudet, "Impedance based combination of visual and force control," in Robotics and Automation, 1998. Proceedings. 1998 IEEE International Conference on, vol. 2. IEEE, 1998, pp. 1743-1748.

[17] G. Ferretti, G. Magnani, P. Rocco, F. Cecconello, and G. Rossetti, "Impedance control for industrial robots," in Robotics and Automation, 2000. Proceedings. ICRA'O0. IEEE International Conference on, vol. 4. IEEE, 2000, pp. 4027-4032.

[18] M. S. Erden and T. Tomiyama, "Human-intent detection and physically interactive control of a robot without force sensors," IEEE Transactions on Robotics, vol. 26, no. 2, pp. 370-382, 2010.

[19] M. Prats, P. J. Sanz, and A. P. Del Pobil, "Reliable non-prehensile door opening through the combination of vision, tactile and force feedback," Autonomous Robots, vol. 29, no. 2, pp. 201-218, 2010.

[20] L. Roveda, F. Vicentini, and L. M. Tosatti, "Deformation-tracking impedance control in interaction with uncertain environments," in 2013 IEEE/RSJ International Conference on Intelligent Robots and Systems. IEEE, 2013, pp. 1992-1997.

[21] M. H. Raibert and J. J. Craig, "Hybrid position/force control of manipulators," Journal of Dynamic Systems, Measurement, and Control, vol. 103, no. 2, pp. 126-133, 1981.

[22] M. T. Mason, "Compliance and force control for computer controlled manipulators," IEEE Transactions on Systems, Man, and Cybernetics, vol. 11, no. 6, pp. 418-432, 1981.

[23] T. Yoshikawa, "Dynamic hybrid position/force control of robot manipulators-description of hand constraints and calculation of joint driving force," IEEE Journal on Robotics and Automation, vol. 3, no. 5, pp. 386-392, 1987

[24] N. H. McClamroch and D. Wang, "Feedback stabilization and tracking of constrained robots," IEEE Transactions on Automatic Control, vol. 33, no. 5, pp. 419-426, 1988.

[25] R. K. Kankaanranta and H. N. Koivo, "Dynamics and simulation of compliant motion of a manipulator," IEEE Journal on Robotics and automation, vol. 4, no. 2, pp. 163-173, 1988.

[26] B. Siciliano and L. Villani, "A passivity-based approach to force regulation and motion control of robot manipulators," Automatica, vol. 32, no. 3, pp. 443-447, 1996.

[27] L. Villani, C. C. De Wit, and B. Brogliato, "An exponentially stable adaptive control for force and position tracking of robot manipulators," IEEE Transactions on Automatic Control, vol. 44, no. 4, pp. 798-802, 1999.

[28] T. Yoshikawa, "Force control of robot manipulators," in Robotics and Automation, 2000. Proceedings. ICRA'O0. IEEE International Conference on, vol. 1. IEEE, 2000, pp. 220-226.

[29] J. K. Salisbury, "Active stiffness control of a manipulator in cartesian coordinates," in Decision and Control including the Symposium on Adaptive Processes, 1980 19th IEEE Conference on. IEEE, 1980, pp. 95-100.

[30] N. Hogan, "Impedance control of industrial robots," Robotics and Computer-Integrated Manufacturing, vol. 1, no. 1, pp. 97-113, 1984.

[31] D. E. Whitney, "Historical perspective and state of the art in robot force control," The International Journal of Robotics Research, vol. 6, no. 1, pp. 3-14, 1987.

[32] C. H. An, C. G. Atkeson, and J. M. Hollerbach, Model-based control of a robot manipulator. MIT press, 1988.

[33] R. J. Anderson, "Passive computed torque algorithms for robots," in Decision and Control, 1989., Proceedings of the 28th IEEE Conference on. IEEE, 1989, pp. 1638-1644.

[34] _ - "Dynamic damping control: Implementation issues and simulation results," in Robotics and Automation, 1990. Proceedings., 1990 IEEE International Conference on. IEEE, 1990, pp. 68-77.

[35] P. Chang, D. Kim, and K. Park, "Robust force/position control of a robot manipulator using time-delay control," Control Engineering Practice, vol. 3, no. 9, pp. 1255-1264, 1995.
[36] A. Castaño and S. Hutchinson, "Visual compliance: Task-directed visual servo control," IEEE transactions on Robotics and Automation, vol. 10, no. 3, pp. 334-342, 1994.

[37] B. J. Nelson, J. D. Morrow, and P. K. Khosla, "Improved force control through visual servoing," in American Control Conference, Proceedings of the 1995, vol. 1. IEEE, 1995, pp. 380-386.

[38] K. Hosoda, K. Igarashi, and M. Asada, "Adaptive hybrid control for visual and force servoing in an unknown environment," IEEE Robotics \& Automation Magazine, vol. 5, no. 4, pp. 39-43, 1998.

[39] D. Xiao, B. K. Ghosh, N. Xi, and T. J. Tarn, "Sensor-based hybrid position/force control of a robot manipulator in an uncalibrated environment," IEEE Transactions on Control Systems Technology, vol. 8, no. 4, pp. 635-645, 2000

[40] W.-C. Chang and C.-C. Wu, "Integrated vision and force control of a 3-dof planar robot," in Control Applications, 2002. Proceedings of the 2002 International Conference on, vol. 2. IEEE, 2002, pp. 748-753.

[41] Y. Zhao and C.-C. Cheah, "Hybrid vision-force control for robot with uncertainties," in Robotics and Automation, 2004. Proceedings. ICRA'04. 2004 IEEE International Conference on, vol. 1. IEEE, 2004, pp. 261-266.

[42] T. Olsson, R. Johansson, and A. Robertsson, "Flexible force-vision control for surface following using multiple cameras," in Intelligent Robots and Systems, 2004.(IROS 2004). Proceedings. 2004 IEEE/RSJ International Conference on, vol. 1. IEEE, 2004, pp. 789-803.

[43] H. Xie, L. Chen, L. Sun, and W. Rong, "Hybrid vision-force control for automatic assembly of miniaturized gear system," in Proceedings of the 2005 IEEE International Conference on Robotics and Automation. IEEE, 2005, pp. 1368-1373.

[44] A. C. Leite, F. Lizarralde, and L. Hsu, "Hybrid vision-force robot control for tasks on unknown smooth surfaces," in Proceedings 2006 IEEE International Conference on Robotics and Automation, 2006. ICRA 2006. IEEE, 2006, pp. 2244-2249.

[45] V. Lippiello, B. Siciliano, and L. Villani, "Robot interaction control using force and vision," in 2006 IEEE/RSJ International Conference on Intelligent Robots and Systems. IEEE, 2006, pp. 1470-1475.

[46] R. Smits, H. Bruyninckx, W. Meeussen, J. Baeten, P. Slaets, and J. Schutter, "Model based position-force-vision sensor fusion for robot compliant motion control," in Proc. IEEE Int. Conf. on Multisensor Fusion and Integration for Intelligent Systems, 2006, pp. 501-506.

[47] R. Volpe and P. Khosla, "Experimental verification of a strategy for impact control," in Robotics and Automation, 1991. Proceedings., 1991 IEEE International Conference on. IEEE, 1991, pp. 1854-1860.

[48] Y. Zhou, B. J. Nelson, and B. Vikramaditya, "Fusing force and vision feedback for micromanipulation," in Robotics and Automation, 1998 Proceedings. 1998 IEEE International Conference on, vol. 2. IEEE, 1998, pp. 1220-1225.

[49] R. Jamisola, M. H. Ang, D. Oetomo, O. Khatib, T. M. Lim, and S. Y. Lim, "The operational space formulation implementation to aircraft canopy polishing using a mobile manipulator," in Robotics and Automation, 2002. Proceedings. ICRA'02. IEEE International Conference on, vol. 1. IEEE, 2002, pp. 400-405.

[50] O. Alkkiomaki, V. Kyrki, H. Kalviainen, Y. Liu, and H. Handroos, "Smooth transition from motion to force control in robotic manipulation using vision," in 2006 9th International Conference on Control, Automation, Robotics and Vision. IEEE, 2006, pp. 1-6.

[51] A. Cherubini, R. Passama, P. Fraisse, and A. Crosnier, "A unified multimodal control framework for human-robot interaction," Robotics and Autonomous Systems, vol. 70, pp. 106 - 115, 2015. [Online]. Available: http://www.sciencedirect.com/science/article/ pii/S0921889015000391

[52] J. Baeten and J. De Schutter, "Hybrid vision/force control at corners in planar robotic-contour following," IEEE/ASME Transactions on mechatronics, vol. 7, no. 2, pp. 143-151, 2002.

[53] B. Ulrich, A. Srivastava, and M. Elbestawi, "Analysis of the robotic disc grinding process," The International Journal of Advanced Manufacturing Technology, vol. 7, no. 2, pp. 82-92, 1992.

[54] W. Khalil and E. Dombre, Modeling, identification and control of robots. Butterworth-Heinemann, 2004.

[55] K. M. El Dine, C. Yang, T. Nozaki, and T. Murakami, "Novel walking assist device based on generic human motion tracking criteria," in Advanced Motion Control (AMC), 2016 IEEE 14th International Workshop on. IEEE, 2016, pp. 38-43.

[56] R. Singh, "Grinding and finishing," class notes for Manufacturing Processes, Indian Institute of Technology, Bombay, no date. 